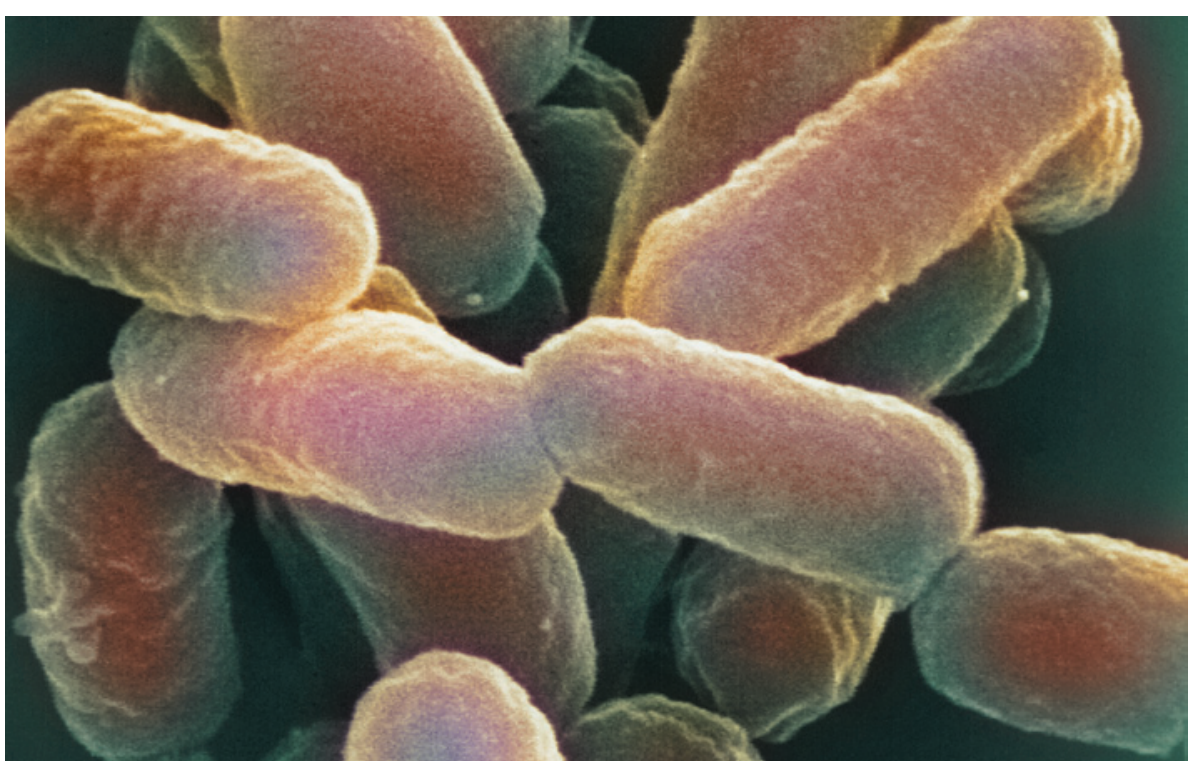

The bacterium Escherichia coli 0157:H7 can cause serious cases of food poisoning.

\title{
FOOD SAFETY
}

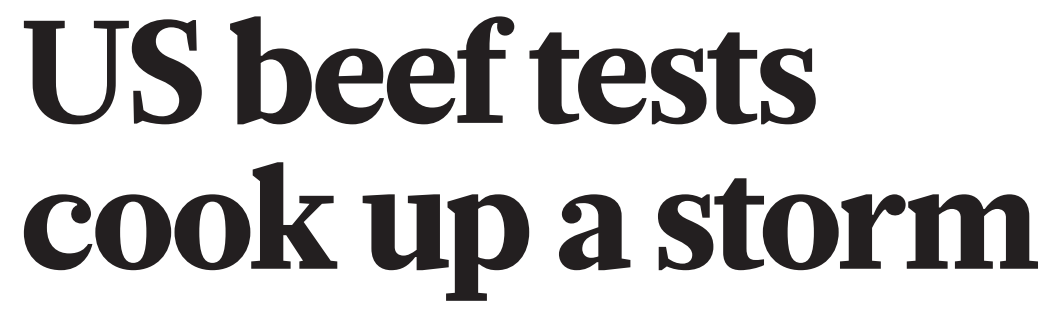

\section{Critics question benefits of broader E. coli screening.}

\section{BY HELEN SHEN}

$\mathrm{E}$ very week, dozens of kilograms of raw beef from meat-processing plants across the United States are rushed to one of three federal laboratories to be picked apart, pulverized, incubated and analysed. Working for the US Department of Agriculture (USDA), technicians in these labs are searching for Escherichia coli O157:H7, a toxic variety of the bacterium that has been monitored in US beef since 1994. But beginning on 4 June, the search will expand to include six more $E$. coli subtypes or 'serogroups'.

US agriculture secretary Tom Vilsack announced the change last September, saying it would "stop problems before they start". The USDA portrays the measure as a way to reduce risk, but it has not quantified the number of people that would be spared illness by the change. With the new screening protocol now imminent, meat producers are questioning the rationale behind the move and at least one outspoken expert agrees.

"I think it's a fundamentally flawed policy, and it's not based on the best science," says Michael Doyle, a microbiologist and director of the Center for Food Safety at the University of Georgia in Griffin. He says that the USDA risks wasting limited resources chasing bacteria whose effects on human health are still unclear.

Most types of E. coli are harmless or even beneficial, such as those that colonize the healthy human gut. But some, such as O157:H7, can produce Shiga toxin, which can be harmful. The USDA began screening raw beef for this microbe after it was linked to the deaths of four children who ate contaminated hamburgers in 1993. Readily identifiable through rapid screening, O157:H7 can sometimes trigger haemolytic uraemic syndrome, which can

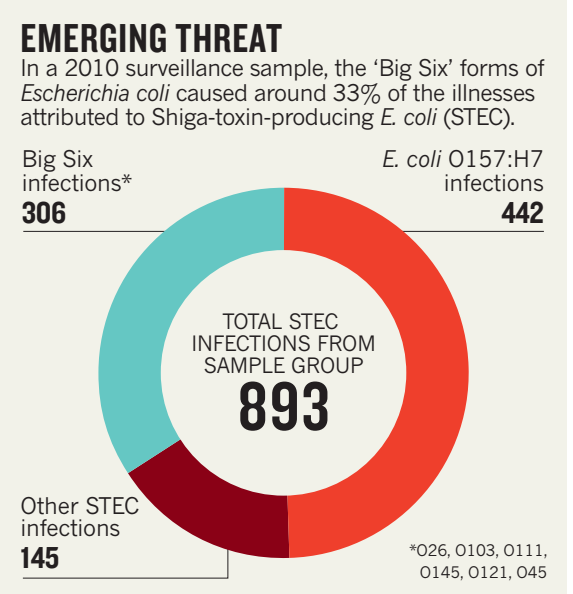

cause life-threatening kidney damage.

Over the past decade, improved diagnostic tests have linked a growing number of foodborne illnesses to other Shiga-toxin-producing E. coli (STEC) serogroups, including the latest additions to the USDA's testing regimen, dubbed the Big Six (see 'Emerging threat'). Last year, an STEC subtype traced to contaminated fenugreek sprouts made thousands of people across Europe ill, and killed at least 53 (see page 559).

The Centers for Disease Control and Prevention (CDC) in Atlanta, Georgia, estimates that 173,000 STEC infections occur annually across the United States, with O157:H7 responsible for roughly $36 \%$ and the Big Six accounting for most of the remainder. By targeting these, the USDA is bringing food regulation in line with public-health data, says Robert Tauxe, deputy director of the division of food-borne, waterborne and environmental diseases at the CDC. "We think this is an important step forward in preventing human disease," he says.

Industry representatives counter that the expanded testing will be redundant, because existing O157:H7 testing is an indicator of overall cleanliness and food safety. "We have done extensive research over the past several years that shows that the preventative controls in our facilities today work equally well for the non-O157:H7 as they do for O157," says James Hodges, executive vice-president of the American Meat Institute (AMI), a trade association in Washington DC.

The AMI estimates that the new tests will cost the industry between US $\$ 172$ million and $\$ 324$ million a year, because it contends the extra sample analysis will force processors to hold onto meat longer before distributing it. More tests will also mean more false positives that require investigation, says Doyle, delaying release of the meat and lowering its market value.

The USDA acknowledges that most infections linked to the Big Six do not stem from contaminated beef but from other sources, such as vegetables. The tests will not eliminate risk of E. coli infection, says David Goldman, assistant administrator for the Office of Public Health Science at the USDA's Food Safety and Inspection Service. Nonetheless, he says, "we expect that this is a truly preventative measure".

The pay-offs of such a precaution are far from certain. Although the Big Six are increasingly seen as sources of illness, their toxic effects are often less severe than those of O157:H7. The serogroups are also divided into strains, not all of which produce toxins. Shiga toxins themselves occur in types that vary in toxicity by up to a factor of 1,000 . Scientists are still trying to determine which strains are likely to produce the most harmful Shiga toxins, and to identify auxiliary molecules that predict a given STEC's virulence in humans.

"We're learning more and more, but it's very 
complex," says Phillip Tarr, a microbiologist at Washington University in St Louis, Missouri. Tauxe concedes that more research is needed to better understand STEC pathology. "This is an area that we expect to evolve," he says. But if the meat-packing industry challenges the rule in court, as it has done for some previous USDA measures, the science of $E$. coli toxicity could soon find itself again under the microscope - this time, a legal one.

\section{Reform falters after Europe's E. coli scare}

\section{Political debate leaves German surveillance law in limbo.}

\section{BY MARIAN TURNER}

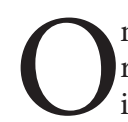
ne year on from Europe's worst recorded outbreak of Escherichia coli infection, governments have made little progress towards improving the monitoring and reporting systems that allowed the crisis to drag on for weeks. The disease, which was spread by contaminated fenugreek sprouts, swept across northern Germany in May and June 2011, infecting thousands and killing 53 people (see Nature 474, 137; 2011). Yet although the panic has sparked some proposed policy changes, these have become mired in political debate at both German and European levels.

Under Germany's current system, it can take up to 18 days for local and state health departments to relay case reports to the Berlinbased Robert Koch Institute (RKI), the German federal agency for disease surveillance. Legislators have proposed a law to bring the country's disease-reporting schedule into line with the World Health Organization's International Health Regulations. The law would require local health authorities to report cases of notifiable diseases to state authorities on the next working day; the states would then have another day to relay the information to the RKI. "We've been waiting almost a decade for this," says Alexander Kekulé, a microbiologist at the Martin Luther University of HalleWittenberg in Halle, Germany.

The draft law has been passed by Germany's federal parliament but is stuck in negotiations at the legislative council that represents Germany's 16 states. For scientists, though, this change would still not be enough. "What really delayed the detection of this outbreak was the irregularity with which patients were referred for microbiological follow-up," says Gérard Krause, an epidemiologist at the RKI. Like many European countries, Germany does not require that a patient with bloody diarrhoea or haemolytic uraemic syndrome (a life-threatening complication of some E. coli infections) be tested for the causative bacterial strain. The same is true of the United States.

After the outbreak, German diagnostic laboratories were provided with kits to test samples for genes belonging to certain pathogenic strains of bacteria, such as those expressing particular toxins, or proteins involved in adhesion or invasion. But physicians are responsible for requesting the tests, and the cost is not covered by German health-insurance companies. "The problem is mostly getting the money to use these kits," says Angelika Fruth, a microbiologist at the RKI, "and that situation is just the same as before the outbreak."

It is not only people who need to be tested the source of most human infection is contaminated food. In the wake of the outbreak, the European Food Safety Authority concluded that sprouted seeds pose a particular foodsafety concern (EFSA J. 9, 2424-2525; 2011), and recommended that a standardized test for sprouts be developed and adopted across the European Union (EU). But EU member countries are still discussing the proposal, and scientists have yet to develop reliable methods to isolate pathogenic bacteria from seeds or sprouts. Meat can also carry E. coli, but whereas the United States screens meat for disease-causing strains (see page 558), EU countries do not routinely do so, relying instead on farm and slaughterhouse hygiene.

There is room for optimism, however. Flemming Scheutz, head of the World Health Organization Collaborative Centre for Reference and Research on Escherichia and Klebsiella in Copenhagen, believes that after last year's outbreak, physicians are at least more likely to recognize and report food-borne infections. The crisis also coincided with the advent of inexpensive whole-genome sequencing technology, resulting in a glut of new bacterial sequences. Genomics and public-health research groups are now studying how these might help to tackle future outbreaks.

"The push for novelty among testing and surveillance systems will come from scientists rather than politics," says Scheutz. - 